\title{
Congenital nephrotic syndrome: A diagnostic and management dilemma
}

\author{
R Moodley, MB ChB, FCPaed, Cert Nephrology (Paeds); E Naicker, MB ChB, DCH, FCP (Paeds), Cert Nephrology (Paeds); \\ R Bhimma, MB ChB, DCH, FCP (Paeds), MMed, MD, Cert in Nephrology (Paeds), ISN Fellowship
}

Department of Paediatrics and Child Health, School of Clinical Medicine, Nelson R Mandela School of Medicine, University of KwaZulu-Natal, Durban, South Africa

Corresponding author: R Bhimma (bhimma@ukzn.ac.za)

Congenital nephrotic syndrome (CNS) is characterised by heavy proteinuria, hypoproteinaemia and oedema presenting in the first 3 months of life. We present a 27-day-old female patient admitted to the Inkosi Albert Luthuli Central Hospital with CNS; one of twins and HIV exposed but uninfected. The child had a cytomegalovirus (CMV) polymerase chain reaction (PCR) positive result in the urine, confirmed on two separate occasions. The CMV PCR for qualitative testing of CMV DNA was negative and quantitative testing was not done. CMV retinitis and central nervous system involvement were absent. Despite treatment with gancyclovir, the CNS did not improve. Further management required indomethacin followed by unilateral nephrectomy to decrease administration of albumin infusions to control oedema. Unfortunately, due to loss to follow-up, the patient demised from probable sepsis. We discuss the challenges we faced with respect to the diagnosis and management of CNS in a resource-limited setting where transplantation is not readily available.

S Afr J Child Health 2015;9(4):140-141. DOI:10.7196/SAJCH.2015.v9i4.903

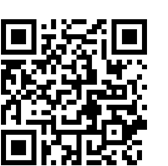

One of the most difficult clinical challenges in the treatment of glomerular diseases in children is the management of congenital nephrotic syndrome (CNS). This is a rare but severe disorder characterised by heavy proteinuria, hypoproteinaemia and oedema presenting in the first 3 months of life. The primary or inherited form is associated most commonly with mutations encoding one of two structured proteins of the slit diaphragm viz. nephrin (NPHS1) and podocin (NPHS2), and a transcription factor (WT1). ${ }^{[1]}$

The secondary form is associated with systemic diseases, typically perinatal infections including congenital syphilis, rubella, toxoplasma, cytomegalovirus (CMV), HIV-1 and hepatitis B. ${ }^{[2]}$ Other less common secondary causes include maternal lupus erythematosus, neonatal antibodies against neutral endopeptidase and drugs such as maternal steroid and chlopheniramine treatment. ${ }^{[3]}$

Treatment with steroids and other immunosuppressive drugs is ineffective in inducing remission. Therefore, the objectives of therapy are to control oedema, delay progression to end-stage kidney disease, prevent and treat complications such as infections and thrombosis, and provide optimal nutrition for growth and development.

The definitive treatment is kidney transplantation once the infant is about $10 \mathrm{~kg}$ in weight. ${ }^{[3]}$ However in resource-limited settings with numerous technical challenges and organ shortage, transplantation is not readily feasible, especially in young recipients.

We present a child with CNS in such a setting and discuss the challenges faced with respect to diagnosis and management. Permission to publish this case as part of a larger study was obtained from the Biomedical Research Ethics Committee of the University of KwaZulu-Natal (Ref number: BE078/13).

\section{Case}

A 27-day-old female patient was admitted to the Inkosi Albert Luthuli Central Hospital in August 2011. The child was one of dizygotic twins delivered at 31 weeks' gestation, with an admission weight of $1.9 \mathrm{~kg}$. There was no history of an enlarged placenta. The mother was infected with HIV-1; serology for syphilis was negative and alphafetoprotein was not tested. She received antiretrovirals antenatally and both infants tested negative for HIV on two separate occasions.

Ten days post delivery, twin 1 presented to a peripheral hospital with anasarca, failure to thrive and clinical sepsis. Laboratory findings showed severe hypoalbuminaemia (serum albumin $18 \mathrm{mg} / \mathrm{dL}$ ), a high urinary protein:creatinine ratio of $2.65 \mathrm{mg} / \mathrm{dL} /$ $\mathrm{mg} / \mathrm{dL}$ (reference range $<2 \mathrm{mg} / \mathrm{dL} / \mathrm{mg} / \mathrm{dL}$ ) with a normal total serum cholesterol of $4.20 \mathrm{mmol} / \mathrm{L}(2.75-4.53 \mathrm{mmol} / \mathrm{L})$ and normal liver enzymes. Kidney function tests showed a serum urea of $4.1 \mathrm{mmol} / \mathrm{L}$ $(1.1-9.1 \mathrm{mmol} / \mathrm{L})$ and serum creatinine of $52 \mu \mathrm{mol} / \mathrm{L}(44-88 \mu \mathrm{mol} / \mathrm{L})$. Thyroid function tests were normal. Kidney ultrasound showed normalsized, hyperechoic kidneys with loss of corticomedullary differentiation. The CMV polymerase chain reaction (PCR) was positive in the urine on two separate occasions. Cardiac echocardiogram showed peripheral pulmonary stenosis while an ophthalmology examination revealed no evidence of CMV retinitis. Cranial ultrasound did not show periventricular calcification. Open renal biopsy was unfortunately not done on the initial admission due to the child being too ill. It was assumed she had CMV-associated CNS in view of the positive CMV PCR in her urine, present before 3 weeks of age. Tests for other infections viz. syphilis, hepatitis B and C and rubella were negative. Due to lack of resources, genetic testing for candidate genes incriminated in the genetic origin of this condition was not possible.

Management included symptomatic control of oedema with albumin infusions. She was also prescribed hydrochlorthiazide, spironolactone, enalapril and a high-protein diet. Indomethacin was initially commenced at a low dose and titrated to a maximum dose. Vitamin and iron supplementation were commenced following control of sepsis. Subsequent testing showed the child to have hyperlipidaemia (serum cholesterol $7.3 \mathrm{mmol} / \mathrm{L}$ and triglycerides $4.35 \mathrm{mmol} / \mathrm{L}$ ) and hypothyroidism with a serum T4 level of $9.1 \mathrm{pmol} / \mathrm{L}$ and thyroidstimulating hormone level of $12.45 \mathrm{mIU} / \mathrm{L}$. She was prescribed simvastatin as well as thyroxine. Anticoagulation therapy was not commenced due to the risk of bleeding, as the platelet count dropped significantly following commencement of indomethacin.

Gancyclovir was given as treatment for CMV for two weeks and she was then discharged on oral valgancyclovir. Valgancyclovir therapy was completed over a 6-month period owing to poor compliance and medication unavailability. Her nutrition was optimised, and she was referred to her base hospital for control of oedema. Weekly monitoring for neutropenia was done during the period of gancyclovir use. She required admissions two to three times a week for albumin infusions In September 2012 an open kidney biopsy showed histopathological features in keeping with the spectrum of CNS. CMV inclusions were 
not seen. She was noted to have severe acute malnutrition with failure to thrive, hepatosplenomegaly and generalised lymphadenopathy. Screening for metabolic diseases, repeat HIV-1 PCR testing, as well as investigations for tuberculosis were negative. Repeated testing for CMV PCR on three occasions was negative.

Despite aggressive nutritional therapy and maximal medical management, she required albumin and diuretic infusions at least two to three times a week, with numerous admissions for lower respiratory tract infections, for which she was treated with bronchodilator nebulisations and intravenous antibiotics. In March 2013, she tested positive for Mycobacterium tuberculosis and received a 6-month course of anti-tuberculosis therapy.

In view of her frequent hospitalisations for control of oedema, a unilateral nephrectomy was performed in October 2013 (age 26 months) to decrease urinary protein losses. The resected left kidney showed dilated Bowman's capsules with florid focal segmental glomerular sclerosis, global glomerulosclerosis and interstitial fibrosis.

Although the child remained mildly oedematous, her albumin and diuretic infusions decreased to not more than once weekly. Her kidney function was normal and she remained euthyroid. Serum albumin levels remained low with an average protein:creatinine ratio of $4.5(\mathrm{mg} / \mathrm{dL} /$ $\mathrm{mg} / \mathrm{dL}$ ). She remained malnourished despite attempts to optimise her nutrition. She had features of global developmental delay; with normal neuroimaging. The child was unfortunately lost to follow-up for about a year and the mother subsequently reported that the child had died, presumably from overwhelming sepsis.

\section{Discussion}

We present a case of a child with CNS, one of twins and HIV-1 exposed but not infected. The case presented both diagnostic and management challenges in a resource-limited setting. Prior to the 1990s, CNS in our region was seen almost exclusively in newborns secondary to congenital syphilis, with a gratifying response to penicillin therapy (personal communication, Adhikari). It is, however, possible that the condition was undiagnosed as it is rare and may have been mistaken for severe acute malnutrition. The latter is not uncommon in our population, where the majority of patients present from a lower socioeconomic background.

The diagnostic dilemma was whether our patient had CNS secondary to CMV or whether the presence of a positive CMV PCR in the urine at just over 3 weeks of age was a gratuitous finding. Screening for CMV involvement of other systems was negative. CMV PCR viral load was not done owing to cost implications. Kidney biopsy did not demonstrate evidence of a proliferative glomerulonephritis, viral immune defects in glomerular cells, or an immune complex glomerulonephritis with IgG, IgA, C3 or $\mathrm{CMV}$ antigens within glomeruli. However, the biopsy was done after gancyclovir treatment. Treatment with an extended course of valganciclovir had no effect on ameliorating the disease.

CMV intrauterine infection is a well-documented cause of the secondary form of this disease. However, the causal relationship between CMV and CNS has been challenged by Frishberg et al., ${ }^{[4]}$ who reported a case of mutated podocin manifesting as CMV-associated CNS and proposed that CMV infection was only a secondary finding. Several reports have established a causal relationship between CMV and CNS..$^{[5]}$ In these reports, CNS was associated with extrarenal manifestations such as neurological, ophthalmological, haematological, cutaneous and pulmonary manifestations. ${ }^{[5]}$ In almost all cases reported to date, CNS associated with CMV responded to treatment. This unfortunately was not the case in our patient, suggesting that CMV infection may have been an incidental finding. This is similar to the case reported by Frishberg et al. ${ }^{[4]}$ in which mutated podocin-associated CNS had CMV as an incidental finding. We unfortunately could not undertake genetic testing in our patient due to lack of resources and financial constraints.

The management of CNS is extremely difficult as immunosuppressive agents are ineffective in alleviating proteinuria and inducing long-term remission. The goals of therapy in the first months of life are to control oedema, prevent progression to end-stage kidney disease, prevent and treat complications such as fluid and electrolyte disturbances, recurrent infections, thrombosis, hypothyroidism, hypertension and hypercholesterolaemia, and optimise nutrition to promote growth and development. When necessary, supplemental calcium and magnesium replacement therapy is given to keep serum levels within normal range. As our patient was able to tolerate adequate amounts of oral feeds to meet dietary requirements, an indwelling nasogastric tube or gastrostomy was not necessary.

Despite maximal antiproteinuric therapy, the patient remained highly proteinuric with failure to thrive. Ideally, bilateral nephrectomies and commencement of dialysis should have been undertaken, but given the challenges faced in our setting, a more conservative approach was adopted and a unilateral nephrectomy was performed to reduce protein losses. This decreased the frequency of albumin infusions while maintaining kidney function. It was hoped that this would allow the child to thrive and gain weight. Only if the child's kidney function had progressed to end-stage kidney disease, would chronic dialysis and a kidney transplant have been considered once an appropriate weight was reached. Unfortunately, sepsis is a major complication of this condition; the child was lost to follow-up and most likely died from overwhelming sepsis.

The only curative treatment of CNS is kidney transplantation. This poses major challenges in resource-limited settings owing to the enormous costs involved, technical challenges and organ shortages. Whereas previously, CNS patients had a higher rate of kidney graft failure compared with patients undergoing transplants for other kidney diseases, particularly in younger recipients $<2$ years old, more recent data have shown graft survival rates of over $80 \%$. This is similar to that obtained with other aetiologies, with 5-year patient survival of $90 \% .{ }^{[6]}$ Although the results of kidney transplantation in these younger patients are improving, chronic allograft nephropathy remains a major problem and a second kidney transplant is inevitable when the patients reach adulthood. ${ }^{[3]}$

\section{Conclusion}

This is the first report of a child with CNS from a resource-limited setting that presented with both a diagnostic and management challenge. Following optimal nutrition and maximum drug therapy, a unilateral nephrectomy was undertaken that ameliorated proteinuria and decreased the need for frequent albumin infusions. We advocate such an approach in a resource-limited setting where access to renal replacement therapy may not be readily available.

Acknowledgments. The authors wish to thank the Medical Manager of Inkosi Albert Luthuli Central Hospital for permission to publish, Ms Nompumelelo Ndlela for her administrative support in formatting the paper, and medical and nursing staff at the various healthcare centres who were involved in the care of the child.

\section{References}

1. Boute N, Gribouval O, Roselli S, et al. NPHS2, encoding the glomerular protein podocin, is mutated in autosomal recessive steroid-resistant nephrotic syndrome. Nat Genet 2000;24(4):349-354. [http://dx.doi.org/10.1038/74166]

2. Holmberg CJH, Tryggavason K, Rapola J. Congenital nephrotic syndrome. In: Barratt TM, Harmon WE, eds. Pediatric Nephrology. 4th ed. Baltimore: Lippincott Williams \& Wilkins, 1999:765-777.

3. Jalanko H. Congenital nephrotic syndrome. Pediatr Nephrol 2009;24(11):21212128. [http://dx.doi.org/10.1007/s00467-007-0633-9]

4. Frishberg Y, Rinat C, Feinstein S, Becker-Cohen R, Megged O, Schlesinger Y. Mutated podocin manifesting as CMV-associated congenital nephrotic syndrome. Pediatr Nephrol 2003;18(3):273-275.

5. Besbas N, Bayrakci U, Kale G, et al. Cytomegalovirus-related congenital nephrotic syndrome with diffuse mesangial sclerosis. Pediatr Nephrol 2006;21(5):740-742. [http://dx.doi.org/10.1007/s00467-003-1079-3]

6. Benfield MR, McDonald RA, Bartosh S, Ho PL, Harmon W. Changing trends in pediatric transplantation: 2001 Annual Report of the North American Pediatric Renal Transplant Cooperative Study. Pediatr Transplant 2003;7(4):321-335. 